\title{
ARTÍCULO 34
}

“Idiomas

El presente Convenio se adopta en idiomas español y portugués, siendo ambos textos igualmente auténticos"

\section{YDANGELY TROPIANO}

Abogada, Especialista en Derecho Laboral y Doctor in Juridical Sciences.

Profesora en las áreas de Salud Ocupacional, Derecho del Trabajo y Administrativo en Instituciones Académicas.

Miembro de Aprendizaje Jurídico, SC.

Venezuela, República Bolivariana de 


\title{
RESUMEN
}

La Seguridad Social tiene como objetivo el desarrollo del trabajo decente, donde el trabajo ha constituido un factor social de fortalecimiento entre las naciones, surgiendo entre países lazos de cooperación en el marco de la globalización, que ha contribuido a la promoción de normativas dirigidas a la protección social de los trabajadores y a su familia. De este modo, en la búsqueda de trabajo decente de trabajadores migrantes y de las personas dependientes de ellos, surge en el año 2007, El Convenio Multilateral Iberoamericano de Seguridad Social, firmada en Santiago Chile, en que el artículo 34, dispone los idiomas que se adopta el convenio, comprendiendo el español y el portugués, donde los ejemplares que se emitan en los referidos idiomas serán igualmente auténticos; no obstante en materia de relaciones internacionales han surgido dilemas en cuanto la interpretación de los tratados o convenios cuyos textos son de dos o más idiomas, de los cuales ante un conflicto se debe aplicar los métodos y principios de interpretación ante la duda en la aplicabilidad de las disposiciones.

PALABRAS CLAVES: Convenio, Seguridad Social, idiomas, interpretación.

\begin{abstract}
Social Security aims at the development of decent work, where work has been a social factor of strengthening among nations, emerging between countries ties of cooperation in the framework of globalization, which has contributed to the promotion of regulations aimed at social protection of workers and their families. Thus, in the pursuit of decent work for migrant workers and those dependent on them, arises in 2007, the Latin American Multilateral Agreement on Social Security, signed in Santiago, Chile, in Article 34, provides languages the agreement is adopted, comprising Spanish and Portuguese, where the copies to be issued in the aforementioned languages are equally authentic; however in international relations have emerged dilemmas regarding the interpretation of treaties or agreements whose texts are of two or more languages, of which a conflict should apply the methods and principles of interpretation in doubt on the applicability of provisions.
\end{abstract}

KEYWORDS: Agreement, Social Security, languages, interpretation 
SUMARIO

I. ASPECTOS GENERALES

II. INTERPRETACIÓN DE LOS CONVENIOS INTERNACIONALES

III. CONCLUSIÓN

e-Revista Internacional de la Protección Social, ISNN 2445-3269. 2017, Vol. II, Nº 1

http://dx.doi.org/10.12795/e-RIPS.2017.i01.12

Página 93 


\section{ASPECTOS GENERALES}

La Seguridad Social tiene como objetivo el desarrollo del trabajo decente, donde el trabajo ha constituido un factor social de fortalecimiento entre las naciones, surgiendo lazos de cooperación en el marco de la globalización, que ha contribuido entre los países a la promoción de normativas dirigidas a la protección social de los trabajadores y a su familia.

Los tratados internacionales tiene una estructura de conformación al igual que las leyes; en este sentido la Organización de las Nacionales Unidas ${ }^{1}$, creo una guía práctica para el diseño de los tratados para aquellos que participan directamente en la celebración de ellos.

En el caso de los tratados o acuerdos que se deposite ante el Secretario de las Naciones Unidas, se concierten únicamente en los idiomas oficiales de las Naciones Unidas, de los cuales predomina el francés o el inglés a diferencia de otros idiomas, a pesar que tampoco es una obligación pero es la preferencia.

En cambio la Secretaria General Iberoamericana, tiene como fin el apoyo de 22 países de los cuales 19 de América Latina (lenguaje castellano y portugués) y los de la Península Ibérica España, Portugal y Andorra $^{2}$, mediante el cual contribuye a la cooperación iberoamericana en el ámbito social, cultural y educativo.

La Secretaría General Iberoamericana, es la organización designada por los miembros de los Estados partes del Convenio Multilateral Iberoamericana de Seguridad Social, para ser depositario.

En el Manual de Tratado indica los postulados para las traducciones, recomendado la utilización de idiomas que sean viables ${ }^{3}$, como se indico el francés o el inglés; no obstante la elección del idioma dependerá de la organización promotora de la normativa, de los cuales se especifica idiomas que sea de gran acceso para los países integrante del convenio o del ámbito de aplicación del mismo.

Es por ello que el Convenio Multilateral Iberoamericano de Seguridad Social contempló el español y el portugués, como idiomas oficiales por ser la mayoría de los Estados partes países donde predomina los referidos idiomas ${ }^{4}$; no obstante es de destacar que cualquier tratado o acuerdo presentado para su registro debe contener una series de requisitos, y entre ellos se destaca los idiomas por las cuales se redacto el acuerdo o tratado.

\footnotetext{
${ }^{1}$ Manual de Tratados, consultado en la página: https://treaties.un.org, en fecha 27-07-2016.

${ }^{2}$ Secretaria General Iberoamericana, consultado en: http://segib.org/quienes-somos/, en fecha 27-07-2016.

${ }^{3}$ Ibidem; pp. 11-12.

${ }^{4}$ Español y portugués. Igual se observa en el artículo 10 del Convenio Iberoamericano sobre el Uso de la Videoconferencia en la Cooperación Internacional entre Sistemas de Justicia, celebrada 3-12-2010; implementándose los idiomas del español y portugués.
}

e-Revista Internacional de la Protección Social, ISNN 2445-3269. 2017, Vol. II, N ${ }^{\circ} 1$

http://dx.doi.org/10.12795/e-RIPS.2017.i01.12

Página 94 
Es de acotar, que el Reglamento de la Corte Internacional de Justicia ${ }^{5}$, establece lo referente a los idiomas, cuando se pretenda introducir una denuncia, indicando lo siguiente:

1. Si las partes acuerdan que todo el procedimiento escrito se siga en uno de los idiomas oficiales de la Corte, los alegatos escritos se presentarán únicamente en ese idioma. A falta de tal acuerdo, todo alegato escrito, o parte de un alegato escrito, se presentará en uno u otro de los idiomas oficiales.

2. Si de acuerdo con el párrafo 3 del Artículo 39 del Estatuto, se usase un idioma distinto del francés o del inglés, se acompañará al original de cada alegato escrito una traducción en francés o en inglés, certificada como exacta por la parte que la presente.

3. Cuando un documento anexo a un alegato escrito no esté redactado en uno de los dos idiomas oficiales de la Corte, se acompañará una traducción en uno de esos dos idiomas, certificada como exacta por la parte que lo presente. La traducción podrá limitarse a parte del anexo o a extractos del mismo pero, en este caso, deberá ir acompañada de una nota explicativa indicando los pasajes traducidos. La Corte podrá, sin embargo, pedir que se facilite la traducción de otros pasajes o una traducción completa. (sic)

Como se desprende, el uso de los idiomas también está configurado para los procesos de denuncia, cuando exista por ejemplo lesión de los derechos, diferencias de interpretación del convenio, entre otras situaciones; de los cuales hay unos parámetros de procedencia cuando estamos enfrente de una denuncia y en especial sobre el tema de varios idiomas, predominando el acuerdo, pero cuando no exista conceso se determinará que el idioma a utilizar son los que están establecido como oficiales; siendo que el escrito que no esté formalizado en algunos de los idiomas se anexará su respectiva traducción.

De este modo, el empleo de varios idiomas no está enfocado solamente para convenios internacionales, sino también para los procedimientos de denuncias, y por ello ante la duda de la interpretación habrá la opción de la traducción.

En este sentido, los tratados, convenios o acuerdos se pactan en más de un idioma, que constituyen lo denominado "idiomas auténticos" 6 ; que son los idiomas que determina el significado, interpretación de sus disposiciones; el problema que surge en la práctica y en especial en materia internacional es la dilucidación de las cláusulas ante un país que su idioma oficial no es aquel contemplado en el convenio y exista duda en su aplicación.

\footnotetext{
${ }^{5}$ Artículo 51, consultado en: http://www.icj-cij.org/homepage/sp/icjrules.php, en fecha 27-07-2016.

${ }^{6}$ Ibidem, p. 66

e-Revista Internacional de la Protección Social, ISNN 2445-3269. 2017, Vol. II, Nº 1

http://dx.doi.org/10.12795/e-RIPS.2017.i01.12 


\section{INTERPRETACIÓN DE LOS CONVENIOS INTERNACIONALES}

En la práctica, la disyuntiva se presenta ante la interpretación de tratados o convenios con idiomas distintos al español, en virtud del significado de las palabras, que a lo mejor para un idioma como el español una palabra tiene un significado distinto para el caso del inglés, francés, portugués, entre otros.

Los tratados autenticados por varios idiomas, su interpretación busca la esencia que quisieron darles las partes al trato, en este sentido NOVAK TALAVERA ${ }^{7}$, indica que los tratados pueden ser interpretados por varios sujetos o entidades, atribuyéndose diversos efectos jurídicos. De este modo, los tratados internacionales pueden ser interpretados por los Estados Partes, metodología esta conocida como interpretación auténtica, cuyos efectos jurídicos no se centra a los Estados partes sino que también es oponible ante terceros.

Ahora bien, la interpretación puede surgir ante un órgano jurisdiccional internacional, cuando surja controversias entre los Estados; cuyo efecto jurídico surtirá entre las partes; no obstante NOVAK TALAVERA ${ }^{8}$ expresa que los tratados también pueden ser interpretados por los diversos órganos de un Estado, estando presente ante un análisis unilateral, de los cuales los efectos jurídicos surtirá en el derecho interno, no siendo oponible a los demás Estados que forman parte del tratado; sin embargo destaca que está metodología igual es extensible en los casos de interpretaciones que realicen las organizaciones internacionales.

Ahora bien, por doctrina y jurisprudencia de la Corte Internacional de Justicia se ha establecido unas reglas para la interpretación de los tratados internacionales, en todo ámbito del derecho internacional; comprendiendo el basamento de la Convención de Viena sobre Derechos de los Tratados.

En este sentido, NOVAK TALAVERA ${ }^{9}$, menciona algunas sentencias de la Corte Internacional de Justicia, en que han consagrado que el artículo 31 de la Convención de Viena sobre Derechos de los Tratados, dispone que un tratado debe ser interpretado de buena fe, y al respecto la Convención señala:

“(...) Regla general de interpretación. I. Un tratado deberá interpretarse de buena fe

\footnotetext{
${ }^{7}$ Novak Talavera, F.; "Los Criterios para la Interpretación de los Tratados". Revista de Derecho. THEMIS. 63; pp. 70-72

${ }^{8}$ Ibidem; p.73

${ }^{9}$ Corte Internacional de Justicia. "Asunto sobre la diferencia territorial entre Libia y Chad". 1994. p. 22. En el mismo sentido véase: Corte Internacional de Justicia. "Asunto de las plataformas petroleras entre Irán y Estados Unidos". 1996. p. 812; Corte Internacional de Justicia. "Asunto de la isla Kasikili/Sedudu entre Botswana y Namibia". 1999. p. 18; Corte Internacional de Justicia. "Asunto LaGrand". 2001. párrafo 99; Corte Internacional de Justicia. "Asunto relativo a la soberanía sobre Pulau Litigan y Pulau Sipadan". 2002. p. 645; Corte Internacional de Justicia. "Asunto Avena y otros nacionales mexicanos". 2004. p. 48; Corte Internacional de Justicia. "Consecuencias jurídicas de la edificación de un muro en el territorio palestino". 2004 p. 174; Corte Internacional de Justicia. "Asunto relativo a la aplicación de la Convención sobre el Genocidio". 2007. párrafo 160.
}

e-Revista Internacional de la Protección Social, ISNN 2445-3269. 2017, Vol. II, Nº 1

http://dx.doi.org/10.12795/e-RIPS.2017.i01.12

Página 96 
conforme al sentido corriente que haya de atribuirse a los términos del tratado en el contexto de estos y teniendo en cuenta su objeto y fin" ${ }^{\prime \prime}$.

Conforme lo antes citado, el principio de interpretación de un tratado o convenio es la buena fe, en base al sentido que hay que darse a los términos de tratado y teniendo en consideración el objeto, la finalidad y la naturaleza del tratado.

Asimismo, NOVAK TALAVERA indica que el Tribunal Europeo de los Derechos Humanos $^{11}$ se ha pronunciado de acuerdo al mismo sentido sobre el método de interpretación de los tratados en el caso "Asunto Golder v. United Kingdom"12.

Aguirre Arango ${ }^{13}$, expresa que la Convención de Viena sobre Derecho de los Tratados, consagra criterios objetivos para la interpretación de un tratado internacional, mencionando la buena fe; el sentido corriente que tiene sus términos (interpretación gramatical semántica), considerando el contexto (interpretación gramatical sintáctica e sistemática), y considerando su objeto y fin (interpretación teológica o finalista).

De este modo, la Convención de Viena sobre Derecho de los Tratados, consagra en el artículo 31, además de la regla principal otro modo de interpretación de los tratados que comprende, además del texto, su preámbulo y anexos, de la siguiente forma:

a) todo acuerdo que se refiera al tratado y haya sido concertado entre todas las partes con motivo de la celebración del tratado;

b) todo instrumento formulado por una o más partes con motivo de la celebración del tratado y aceptado por las demás como instrumento referente al tratado;

3. Juntamente con el contexto, habrá de tenerse en cuenta:

a) todo acuerdo ulterior entre las partes acerca de la interpretación del tratado o de la aplicación de sus disposiciones:

b) toda práctica ulteriormente seguida en la aplicación del tratado por la cual conste el acuerdo de las partes acerca de la interpretación del tratado:

\footnotetext{
${ }^{10}$ Viena, 23 de mayo de 1969.

${ }^{11}$ El Tribunal Europeo de los Derechos Humanos, es quien supervisa que los Estados partes cumplan con los tratados sobre derechos humanos. Sus decisiones son de carácter vinculante para los miembros del Consejo de Europa, de los cuales su incumplimiento conlleva la expulsión del Estado parte. El Tribunal Europeo se ocupa de los casos en los que el particular no ha recibido una compensación adecuada por la violación de alguno de sus derechos en los tribunales de su país o no ha podido acceder al sistema nacional de justicia. Asimismo el Tribunal desempeña una función asesora que le permite elaborar opiniones consultivas; y tratan más a fondo un artículo o un aspecto concreto de un Convenio para ayudar a interpretar su significado. Consultado en:

https://www.crin.org/es/guias/onu-sistema-internacional/mecanismos-regionales/tribunal-europeo-dederechos-humanos, en fecha 20-08-2016.

${ }^{12}$ Serie A, 1995.

${ }^{13}$ Aguire Arrango, J.P.; La Interpretación de la Convención Americana sobre Derechos Humanos; p.73.
}

e-Revista Internacional de la Protección Social, ISNN 2445-3269. 2017, Vol. II, Nº1

http://dx.doi.org/10.12795/e-RIPS.2017.i01.12

Página 97 
c) toda forma pertinente de derecho internacional aplicable en las relaciones entre las partes.

4. Se dará a un término un sentido especial si consta que tal fue la intención de las partes.

De este modo, el artículo 31 de la Convención, consagra varias técnicas de interpretación de los cuales se tendrá en cuenta el texto, el prologo o introducción y los anexos del tratado; sin embargo cuando el texto del tratado es claro se hace fluido el análisis hermenéutico, partiendo de lo intrínseco a lo extrínseco.

NOVAK TALAVERA señala que la Comisión de Derecho Internacional de la Organización de las Naciones Unidas, determina que el artículo 31 de la Convención al indicar la expresión regla en singular y no en plural hace referencia que los principios de interpretación deben ser aplicados de forma integral y en conjunto ${ }^{14}$.

Por otra parte la Convención establece otros métodos de interpretación, en este sentido en el artículo $32^{15}$, consagra el modo complementario que consiste en la utilización de trabajos realizados de forma previa al tratado y el análisis de las condiciones por las cuales surge la celebración del mismo. Esta técnica se emplea cuando se requiere confirmar el sentido de la aplicación de la buena fe, que consagra el artículo 31, o cuando el texto presente lagunas, vacios o un resultado irrazonable o absurdo.

A pesar de estos procesos de interpretación, no podemos dejar a un lado, cuando tenemos tratados autenticados en dos o más idiomas, que consiste la forma de análisis que contempla el artículo $33^{16}$ de la Convención; el cual deriva cuatro métodos, siendo que el primero se relaciona al artículo 31 de la Convención, donde prevalecerá la buena fe en cada texto con su idioma, salvo que el tratado establezca o las partes hayan convenido que en caso de desacuerdos prevalecerá uno de los textos y no ambos. El

\footnotetext{
${ }^{14}$ Novak Talavera, F.;"Los Criterios para la Interpretación de los Tratados”. Op. cit.; p.74.

${ }^{15} 32$. Medios de interpretación complementarios. Se podrán acudir a medios de interpretación complementarios, en particular a los trabajos preparatorios del tratado y a las circunstancias de su celebración, para confirmar el sentido resultante de la aplicación

del artículo 31, o para determinar el sentido cuando la interpretación dada de conformidad con el artículo 31:

a) deje ambiguo u oscuro el sentido; o

b) conduzca a un resultado manifiestamente absurdo o irrazonable.

16 33. Interpretación de tratados autenticados en dos o más idiomas.

1. Cuando un tratado haya sido autenticado en dos o más idiomas, el texto hará igualmente fe en cada idioma, a menos que el tratado disponga o las partes convengan que en caso de discrepancia prevalecerá uno de los textos.

2. Una versión del tratado en idioma distinto de aquel en que haya sido autenticado el texto será considerada como texto auténtico únicamente si el tratado así lo dispone o las partes así lo convienen.

3. Se presumirá que los términos del tratado tienen en cada texto auténtico igual sentido.

4. Salvo en el caso en que prevalezca un texto determinado conforme a lo previsto en el párrafo 1,. cuando la comparación de los textos autenticas revele una diferencia de sentido que no pueda resolverse con la aplicación de los artículos 31 y 39, se adoptará el sentido que mejor concilie esos textos, habida cuenta del objeto y fin del tratado.
}

e-Revista Internacional de la Protección Social, ISNN 2445-3269. 2017, Vol. II, Nº 1

http://dx.doi.org/10.12795/e-RIPS.2017.i01.12

Página 98 
segundo punto, contempla que solo la versión de un tratado basado en un idioma distinto al aquel que fue autenticado, será considerada como texto autentico, siempre que haya sido dispuesto en el tratado o hubiera sido convenido por las partes.

En el tercer caso, simplemente no habrá diferencias entre los textos con su idioma, porque se tomara que los términos del tratado tendrá en cada texto autentico igual sentido; y por último es en la situación que ante un análisis comparativo de los textos autenticados se verificare diferencia de sentido, que no puede ser subsanado o resuelto conforme los artículos 31 y 39, se adoptara el sentido que mejor resulte de los textos, considerando el objeto y el fin del tratado.

Ahora, en el Convenio Multilateral Iberoamericano de Seguridad Social dispone como idiomas del texto el español y portugués, los cuales en principio no debería existir confrontación de interpretación del convenio, en virtud que la lengua común de los países miembros en su mayoría es el castellano, español y portugués; sin embargo para estos países podría existir diferenciación de significado de ciertas palabras que podría variar el significado de una frase.

La utilización de estos dos idiomas, se extendió en el Acuerdo de Aplicación del Convenio Multilateral Iberoamericano de Seguridad Social ${ }^{17}$, para ambos textos igualmente auténticos.

A este punto es de hacer referencia, que el Convenio Multilateral Iberoamericano de Seguridad Social se enfoca a la protección de derechos sociales y económicos, cuyo objeto es proteger a los trabajadores migrantes y las personas que dependa de ellos, con sus exclusiones; con el fin que puedan disfrutar de los beneficios generados por el trabajo realizado en los países Iberoamericanos receptores; resultando que en caso de existir dudas en la aplicación del Convenio, por relucir diferencias interpretativas, se debe utilizar la gama de métodos que facilita el Convenio de Viena adoptado por doctrina y jurisprudencia.

Siendo además, que la interpretación debe ser cónsono con los principios del derecho de trabajo establecido en el ámbito internacional, adoptados en las legislaciones internas en los Estados partes, dirigida a lo que mejor beneficie al trabajador y que le garantice de igualdad condiciones el disfrute el derecho que le asiste, sin apartarnos como se indico de utilizar los métodos de interpretación en los casos que se requiera cuando estamos ante convenios con idiomas distintos.

\section{CONCLUSIÓN}

De lo expuesto antes podemos determinar, que por doctrina y jurisprudencia se ha establecido las técnicas para la interpretación de tratados internacionales, cuya concepción se ha extendido también a los convenios, en virtud de lo dispuesto por la Convención de Viena sobre Derechos de los Tratados.

\footnotetext{
${ }^{17}$ Hecho en Lisboa el 11-09-2009, aplicación provisional, artículo 36, consultado en: http://www.segsocial.es/Internet_1/Normativa/147178, en hecha 21-08-2016.
}

e-Revista Internacional de la Protección Social, ISNN 2445-3269. 2017, Vol. II, Nº 1 
Es de destacar, que el uso de varios idiomas se configura también en los procesos de denuncias, empleando en principio aquellos contemplados por acuerdo pero ante la ausencia de ello, y ante la duda de su interpretación en los argumentos el uso de la traducción.

Que a pesar que existe dilemas, de la forma cómo deben ser interpretados los convenios o tratados, cuando su texto autenticado sea emitido por dos o más idiomas diferentes, de los cuales puede originarse significados distintos en palabras que tienda a desvirtuar el objeto o la intención del convenio o tratado; no obstante ante esta situación ha prevalecido la interpretación de la buena fe, considerándose en caso de persistir dudas o ambigüedades, el estudio de trabajos previos a la celebración del convenio, el análisis de la naturaleza, objeto y fin del sentido del convenio.

Esta metodología trata de evitar, confusión en la validez de las interpretaciones por los Estados parte, porque siempre existirá la apreciación individual, subjetiva y unilateral; como sucedió en una oportunidad que a España se le concedió la declaración interpretativa; hecho distinto que surgió con el caso Telmestasch Suiza, que la Comisión de los Derechos Humanos sostuvo la competencia para conocer de las reservas por ser de carácter objetivo las obligaciones asumidas por los Estados partes ${ }^{18}$.

Cuando estamos ante tratados o convenios en materia laboral, que aborda la seguridad social, en que el protagonista es el trabajador en compañía de su grupo familiar, debe imperarse otras reglas o principios de interpretación, en virtud que está en juego la protección social y económica de aquella persona que está ante una situación de vulnerabilidad al hacer desprendida de su país de origen.

En el caso del Convenio Multilateral Iberoamericano de Seguridad Social, en que se plasmo como idiomas el español y portugués, el texto en principio no debería transmitir discrepancia de interpretación, por motivo que la lengua común de los Estados partes miembros de la organización iberoamericana es el español, castellano y portugués; sin embargo no podemos obviar que en la práctica podría en algún momento relucir entre ellos alguna diferencia de interpretación sea del Convenio Marco o de aquellos convenios o acuerdos específicos que se desarrolle de esté, porque siempre habrá una palabra que comunique un significado distinto a lo que realmente se quiere, lo cual esto se evita cuando estos instrumentos pasa por su revisión por los Estados partes ante de su ratificación, aprobación o aceptación.

De ser el caso, que surja alguna discrepancia, laguna o disquisición producto de las palabras emitidas en sus diferentes idiomas, debe tenerse por norte los métodos de interpretación establecido en la Convención de Viena sobre Derechos de los Tratados, abarcándose a su vez el estudio de los principios protectores reconocidos por el derecho internacional laboral que los Estados partes han incorporado en sus legislaciones internas; como son la aplicación de la norma que más favorezca y protege al trabajador; la progresividad e indivisibilidad de los derechos, la eficacia en su aplicación sin

\footnotetext{
${ }^{18}$ Carrillo Salcedo, J.A.; El Convenio Europeo de Derechos Humanos y sus Protocolos Adicionales, consultada en: http://www.corteidh.or.cr/tablas/r11953.pdf, en fecha 21-08-2016, p-p.14-16.
}

e-Revista Internacional de la Protección Social, ISNN 2445-3269. 2017, Vol. II, Nº1

http://dx.doi.org/10.12795/e-RIPS.2017.i01.12

Página 100 
restricciones o limitaciones en el goce; sin que ello tampoco extienda a lo expresamente dispuesto en el convenio. 